\title{
A SEPARATED SPLITTING TECHNIQUE FOR DISCONNECTED RARE EVENT SETS
}

\author{
Wander Wadman \\ Daan Crommelin \\ Jason Frank \\ CWI Amsterdam \\ Science Park 123 \\ Amsterdam, 1098 XG, NETHERLANDS
}

\begin{abstract}
A key challenge for an efficient splitting technique is defining the importance function. If the rare event set consists of multiple separated subsets this challenge becomes bigger since the most likely path to the rare event set may be very different from the most likely path to an intermediate level. We propose to mitigate this problem of path deviation by estimating the subset probabilities separately using a modified splitting technique. We compare the proposed separated splitting technique with a standard splitting technique by estimating the probability of entering either of two separated intervals on the real line. The squared relative error of the estimator is shown to be significantly higher when using standard splitting than when using separated splitting. We show that this difference increases if the rare event probability becomes smaller, illustrating the advantage of the separated splitting technique.
\end{abstract}

\section{INTRODUCTION}

Efficient estimation of very low probabilities is desired in many application areas like telecommunication networks and power grid reliability (de Boer 2000, Wang et al. 2011). Monte Carlo simulation is a robust and popular technique in case the model does not allow an exact derivation, but Crude Monte Carlo may require prohibitively many samples to be practically feasible. To reduce the computational burden, rare event simulation techniques have been developed (Rubino and Tuffin 2009), and (importance) splitting is a well-known variant. A splitting technique aims to reduce the squared relative error by replicating (splitting) trajectories as soon as the rare event is presumed more likely to occur (L'Ecuyer et al. 2006, Glasserman et al. 1999, Villén-Altamirano and Villén-Altamirano 2006).

The efficiency of a splitting technique depends strongly on the choice of the importance function. The optimal importance function strictly increases with the probability of reaching the rare event set from the current state. In this paper we assume no knowledge of this probability except that the underlying Markov process is (the discretization of) a continuous stochastic process. In a simple case with a one-dimensional state space and an interval as the rare event set, any importance function that measures the proximity of the current state to the interval is optimal. In many other cases however, the choice for the importance function is much more difficult.

For example, a challenge arises when the rare event set is disconnected; that is, it consists of multiple nonempty, separated subsets. Such a rare event set is plausible in case of a reliability assessment of a multiple component system for which various combinations of component behavior may lead to a system failure (Grainger and Stevenson 1994, Billinton and Li 1994). One example involves the reliability estimation of an electrical power grid with nodal power injections modeled as stochastic processes (Wadman et al. 2013). Then if at any grid node the injection is very different from that at an adjacent node, the current through the connection in between may exceed its allowed maximum. Hence there could be various combinations of power injections leading to the rare event of a grid fault, and they could involve opposite extremes of 
power injections. In case of a disconnected rare event set an importance function based on the proximity to the complete rare event set may lead to poor results: the algorithm may replicate sample paths near a subset that has only little or even negligible contribution to the rare event probability. Therefore the importance function may be far from optimal as the simulation focuses on areas where the rare event probability is hardly increased or even decreased.

In this paper we assume that we know a partition of the rare event set into subsets that are (or at least might be) separated from each other subset. We propose to estimate the rare event subset probabilities separately. To estimate each subset probability we use a modified splitting technique, taking care that sample paths hitting multiple subsets are not counted multiple times. We introduce notation and the standard splitting framework in Section 2. We discuss properties of the optimal importance function in Section 3. In Section 3.1 we show a one-dimensional problem where the choice for the importance function is clear, and we describe a technique to calibrate the levels using a pilot run. In Section 3.2, we discuss the challenge of a disconnected rare event set, and we describe how to estimate each subset probability separately. We present the numerical results of an experiment in Section 4, after which conclusions are given in Section 5.

\section{SETTINGS AND NOTATION}

\subsection{Rare Event Simulation}

We consider the class of rare events where a discrete-time Markov chain $\left\{X_{t}, t \geq 0\right\}$ with state space $\mathcal{X}$ enters the rare event set $B \subset \mathcal{X}$ before stopping time $\zeta$. We define

$$
\tau_{B}=\inf \left\{t>0: X_{t} \in B\right\}
$$

as the first entrance time in $B$ and $x_{0} \notin B$ as the initial state. We want to estimate the rare event probability

$$
\gamma:=\mathbb{P}\left(\tau_{B}<\zeta \mid X_{0}=x_{0}\right)
$$

Two typical choices for $\zeta$ are a fixed finite time $\zeta=T$ and stopping time $\zeta=\tau_{A}:=\inf \{t>0: \exists 0 \leq s<$ $t$ s.t. $X_{s} \notin A$ and $\left.X_{t} \in A\right\}$, the first time the chain reenters $A$, where $A \subset \mathcal{X}$ is some set that contains $x_{0}$. The Crude Monte Carlo (CMC) estimator

$$
\tilde{\gamma}_{n}:=\frac{1}{n} \sum_{i=1}^{n} \mathbf{1}_{\left\{\tau_{B}<\zeta \text { in sample } i\right\}}
$$

for $\gamma$ is unbiased but its squared relative error

$$
\operatorname{SRE}\left(\tilde{\gamma}_{n}\right):=\frac{\operatorname{Var} \tilde{\gamma}_{n}}{\gamma^{2}}=\frac{\gamma(1-\gamma)}{\gamma^{2} n}=\frac{1-\gamma}{\gamma n}
$$

diverges to infinity as $O(1 / \gamma)$ when $\gamma \rightarrow 0$. Therefore, to estimate a very small probability using CMC simulation, one will need a number of samples that may be computationally too intensive in practice.

\subsection{Importance Splitting}

Any splitting technique starts by defining an importance function $h: \mathcal{X} \mapsto \mathbb{R}$ that assigns an importance value to each chain state. We assume that $A$ and $B$ are determined in terms of this importance function:

$$
A=\{x \in \mathcal{X}: h(x) \leq 0\} \text { and } B=\{x \in \mathcal{X}: h(x) \geq 1\} .
$$

The interval $[0,1]$ is divided into $m$ subintervals with thresholds $0=l_{0}<l_{1}<\cdots<l_{m}=1$. We define $T_{k}=\inf \left\{t>0: h\left(X_{t}\right) \geq l_{k}\right\}$ as the first time of hitting the $k$-th level and $D_{k}=\left\{T_{k}<\zeta\right\}$ as the event that 
the $k$-th level is hit during $[0, T]$. Obviously, $\mathbb{P}\left(D_{0}\right)=1$, and $\mathbb{P}\left(D_{m}\right)=\gamma$ is the value of interest. As $D_{m} \subset D_{m-1} \subset \cdots \subset D_{0}$, we decompose

$$
\gamma=\prod_{k=1}^{m} \mathbb{P}\left(D_{k} \mid D_{k-1}\right)
$$

in $m$ conditional probabilities $p_{k}:=\mathbb{P}\left(D_{k} \mid D_{k-1}\right)$, which we will estimate separately. Generating independent sample paths from the conditional distribution of the entrance state $\left(T_{k-1}, X_{T_{k-1}}\right)$ given $D_{k-1}$ would give us an estimate for $p_{k}$. However, as we do not know this distribution for levels $k>1$, we use its empirical distribution, obtained from samples of the previous level. In this way, we proceed recursively, and at each level $k$ we estimate $p_{k}$ by the proportion $\hat{p}_{k}$ of sample paths for which $D_{k}$ occurs (see Figure 1).

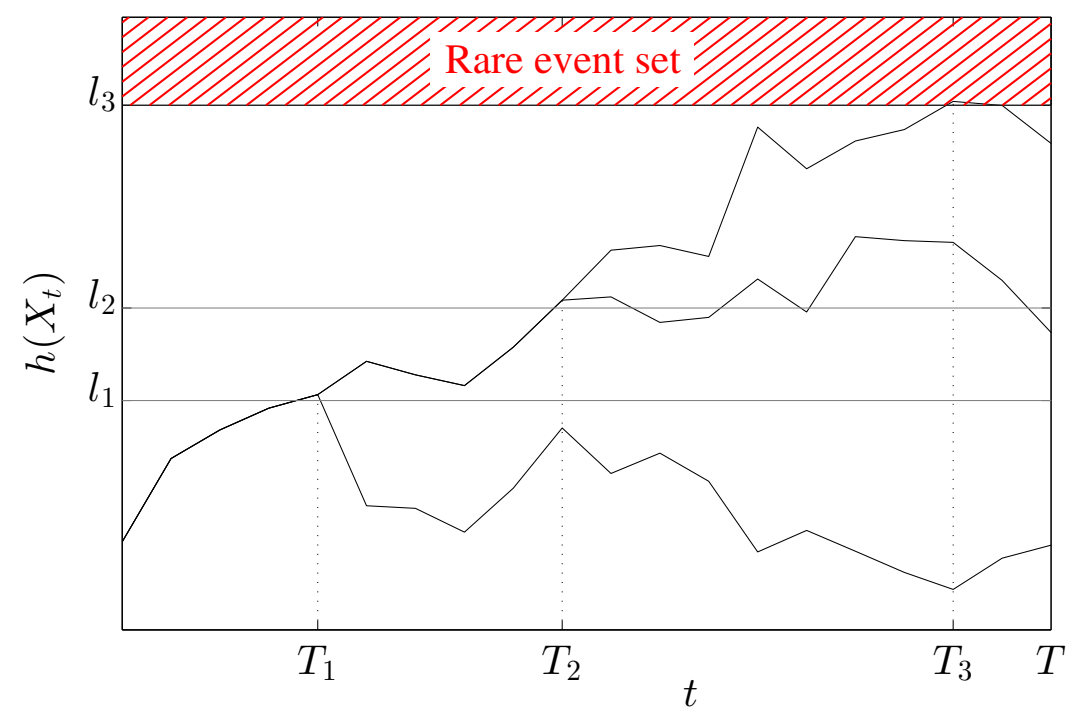

Figure 1: A minimal example of a splitting simulation with $\hat{p}_{1}=1, \hat{p}_{2}=\hat{p}_{3}=1 / 2$.

Then the product

$$
\hat{\gamma}:=\prod_{k=1}^{m} \hat{p}_{k}
$$

is an unbiased estimator for $\gamma$ for several variants of the splitting technique, for example Fixed Splitting, Fixed Effort and Fixed Number of Successes. Proofs that the estimator is unbiased for these three variants can be found in Dean and Dupuis (2009), Del Moral and Garnier (2005) and Amrein and Künsch (2011), respectively. Fixed Splitting determines the number of splittings per level hit in advance. A disadvantage of this technique is that, especially when the number of levels and the thresholds are far from optimally chosen, paths may hit the next level many times or never. The former situation results in a high computational burden, the latter situation in a useless estimate $\hat{\gamma}=0$. Fixed Effort attempts to overcome these issues by fixing the total number of samples per level. Unfortunately, as the realized number of hits may differ from the expected number of hits, the same issues of path explosion and extinction, although to a lesser extent, may persist. Fixed Number of Successes (FNS) fixes the number the number of hits $r_{k}$ for all levels $k$, repeating the simulation at one level until $r_{k}$ hits are observed. This technique avoids the issues of path explosion and extinction by adjusting the computational effort. We use the FNS splitting technique in the rest of this paper. 


\section{FINDING A SUITABLE IMPORTANCE FUNCTION}

Finding a suitable importance function is crucial to reduce the variance of the splitting estimator (Garvels 2000, L'Ecuyer et al. 2006). The importance function should 'reward good behavior' by splitting trajectories that are more likely to hit the rare event set. Glasserman et al. (1998) show that the levels should be chosen in a way consistent with the most likely path to the rare event set. Ideally, this path should coincide with the most likely path to any intermediate level. In case $\zeta=\tau_{A}$, Garvels et al. (2002) propose to use the importance function

$$
g\left(h^{*}(x)\right)
$$

with $g:[0,1] \mapsto \mathbb{R}$ some strictly increasing function and

$$
h^{*}(x):=\mathbb{P}\left(\tau_{B}<\zeta \mid X_{0}=x\right) .
$$

In the field of molecular dynamics $h^{*}(x)$ is called the committor function, or the optimal reaction coordinate, giving the probability that a trajectory initiated in state $x$ will reach first the product state rather than the reactant state (Vanden-Eijnden and Venturoli 2009, Maragliano et al. 2006). As knowing $h^{*}(x)$ would defeat the point of using simulation, the lesson is to find an importance function that is close to (2). Some results on the efficiency of splitting can be derived under the assumption that the probability of hitting the next level does not depend on the current entrance state

$$
\mathbb{P}\left(D_{k} \mid D_{k-1},\left(T_{k-1}, X_{T_{k-1}}\right)\right)=p_{k} \forall\left(T_{k-1}, X_{T_{k-1}}\right) .
$$

Under this assumption and using the optimal choice for $m$ and $p_{k}$ Amrein and Künsch (2011) show that the squared relative error of the FNS estimator is proportional to

$$
\operatorname{SRE}(\hat{\gamma}) \propto \frac{(\log \gamma)^{2}}{n} .
$$

Hence the $\operatorname{SRE}(\hat{\gamma})$ diverges as $O\left((\log \gamma)^{2}\right)$ when $\gamma \rightarrow 0$. This squared logarithmic divergence rate is slower than the divergence rate of the CMC squared relative error in (1). Splitting estimators outperfom the CMC estimator in this sense. Furthermore, under assumption (4) the authors show that $\hat{p}_{1}, \ldots, \hat{p}_{m}$ are independent and derive the bound

$$
\operatorname{SRE}(\hat{\gamma}) \leq-1+\prod_{k=1}^{m} \frac{r_{k}-1}{r_{k}-2}
$$

for the squared relative error of the FNS splitting estimator. That is, the accuracy of the estimator can be controlled by choosing the number of hits $r_{k}$ sufficiently large for all $k$. In Section 4 we will check if the simulation results of an experiment fulfill bound (5). As the validity of this bound is a necessary condition for (4) to hold, this check will serve as a test for the quality of the chosen importance function.

\subsection{A Simple Case with a Connected Rare Event Set}

Consider the one-dimensional case $\mathcal{X}=\mathbb{R}, X_{t}$ the discretization of a continuous stochastic process and $B=[b, \infty)$ an interval for some $b>x_{0}$. Then $\gamma=\mathbb{P}\left(\tau_{B}<\zeta \mid X_{0}=x_{0}\right)$ is a barrier crossing probability and it is fair to assume that $\mathbb{P}\left(\tau_{B}<\zeta \mid X_{0}=x\right)$ increases in $x$ as long as $x<b$, since for the continuous counterpart of $X_{t}$ this is certainly true. Therefore, we may assume that the normalized proximity function

$$
h_{p}(x):=(b-x) /\left(b-x_{0}\right)
$$

is of the form (2) even though $g$ and $h^{*}$ are not explicitly known. Stated in terms of Glasserman et al. (1998), the most likely path to the rare event set will automatically coincide with the most likely path to any intermediate level in this simple case. 


\section{Wadman, Crommelin, and Frank}

The remaining challenge is to find a suitable partition of the interval $[0,1]$ into level thresholds. Amrein and Künsch (2011) propose a pilot run to obtain a rough idea of the optimal number and height of the thresholds for a subsequent final run. That is, they suggest a splitting simulation with a large number of levels and a moderate number of level hits such that the computational intensity is small compared to that of the final run. The authors showed that the optimal value for $p_{k}$ in terms of variance reduction is $p_{\text {opt }}:=0.2032$. Therefore, a pilot estimate of $p_{k}$ close to one suggests to merge level $k$ with a adjacent level, whereas a pilot estimate close to zero suggests to divide the level into multiple levels.

Along these lines we now describe such a calibration procedure in more detail. That is, we search for $m$, and for all $k=1, \ldots, m-1$ we search for the threshold $l_{k}$ such that

$$
p\left(l_{k}\right)=p_{\mathrm{opt}}^{m-k},
$$

where $p(l)$ is the conditional probability of the rare event given that the importance function has exceeded $l$. Assuming no knowledge of $h^{*}$, we perform a pilot run using many levels, say 20, and equidistant thresholds $l_{k}^{\text {pilot }}=k / 20$. As this will yield information on $p(l)$ only at the pilot threshold values $l=l_{i}^{\text {pilot }}$, we assume a power law relationship for $p(l)$ between each pair of subsequent pilot thresholds:

$$
p(\alpha \Delta l)=p(\Delta l)^{\alpha},
$$

for $\alpha>0$. For example, if the top level probability $\hat{p}_{m}^{\text {pilot }}=p_{\text {opt }}^{2}$, then (7) suggests to set the penultimate threshold $l_{m-1}$ twice as close to the top as the penultimate pilot threshold $l_{19}^{\text {pilot }}$. Hence, we approximate $p(l)$ by the log-linear interpolation $p^{*}(l)$ of the estimators

$$
\prod_{i=k+1}^{20} \hat{p}_{i}^{\text {pilot }}
$$

for $p\left(l_{k}^{\text {pilot }}\right)$, as shown in Figure 2. Then we simply solve the obtained piecewise linear equation

$$
\log p^{*}\left(l_{k}\right)=(m-k) \log p_{\text {opt }},
$$

for $l_{k}$. The number of levels is automatically found by computing $l_{m-1}, l_{m-2}, \ldots, l_{i}$ where $i$ is the last index such that $l_{i}>0$. After reindexing we have found the levels $0=l_{0}<\cdots<l_{m}=1$ that we use for the final run.

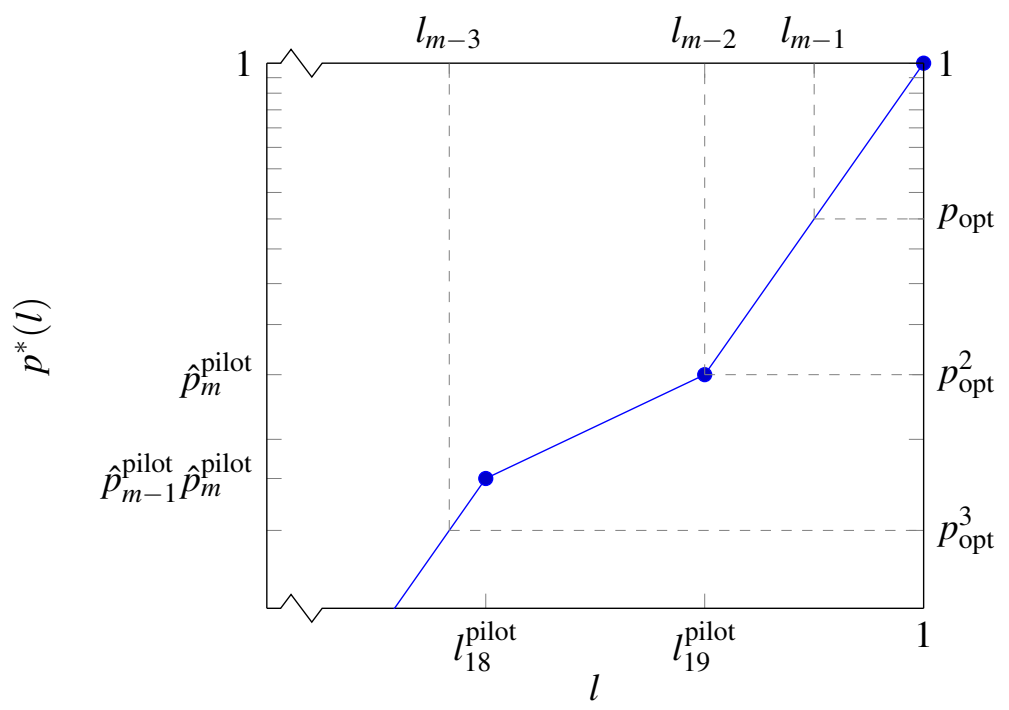

Figure 2: An updated set of thresholds $l_{0}, \ldots, l_{m}$ is found by a log-linear interpolation of the estimates of reaching the rare event set from the pilot thresholds. 


\section{Wadman, Crommelin, and Frank}

\subsection{A Disconnected Rare Event Set}

Now we consider a possibly multidimensional state space $\mathcal{X}$, and a rare event set $B$ that can be partitioned into two nonempty, separated subsets $B_{1}$ and $B_{2}$ (see Figure 3). By an induction argument the rest of this section can be generalized to a partition into more than two subsets.

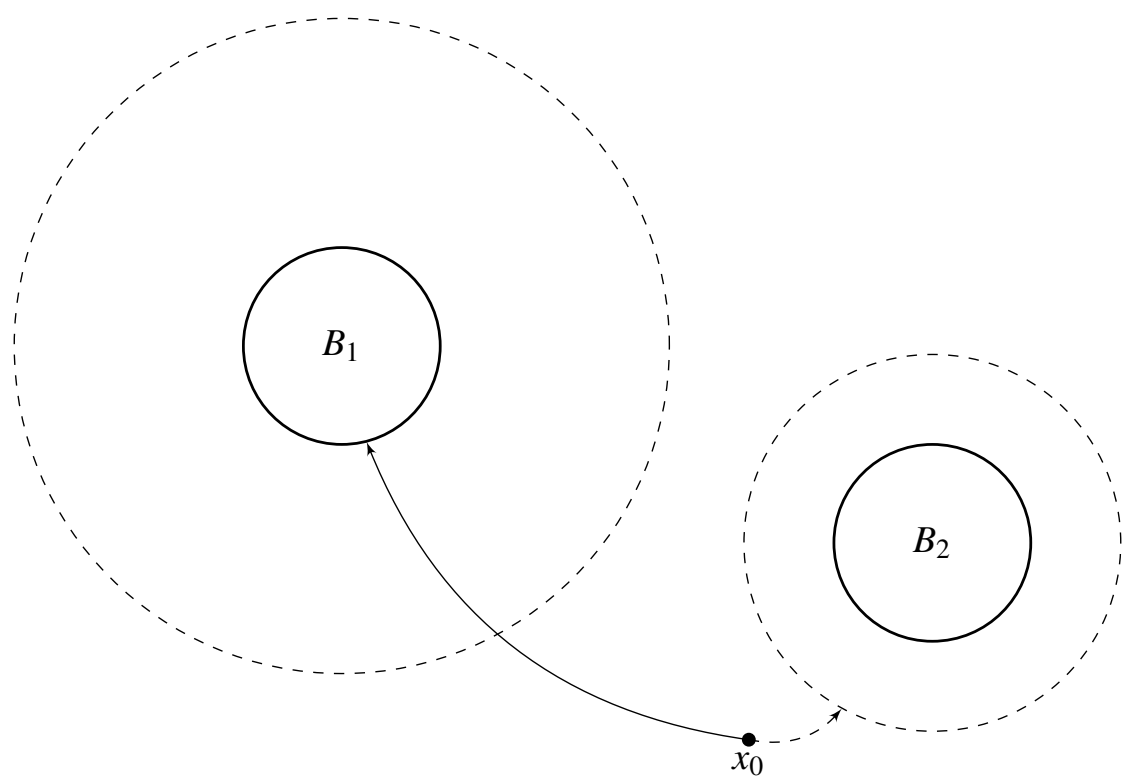

Figure 3: For a general Markov chain, the most likely path to the disconnected rare event set $B=B_{1} \cup B_{2}$ (entering either of the solid circles) may be very different from the most likely path to an intermediate level (entering either of the dashed circles).

As an extension of (6), an intuitive choice for the importance function is the maximum

$$
h_{12}(x):=\max _{i=1,2} h_{i}(x)
$$

of the normalized proximities of $x$ to each subset:

$$
h_{i}(x):=1-\frac{d\left(x, B_{i}\right)}{d\left(x_{0}, B_{i}\right)} .
$$

Here $d(v, V)$ denotes the distance between point $v \in \mathcal{X}$ and set $V \subset \mathcal{X}$. However, as we assume no prior knowledge of $h^{*}(x)$, it could be that the most likely path to an intermediate level approaches $B_{2}$, whereas the most likely path to $B$ does not and instead goes directly to $B_{1}$ (see Figure 3 ). If $X_{t}$ is the discrete counterpart of a continuous stochastic process, we may expect that this deviation between the most likely paths to different level sets becomes smaller as the distance between $B_{1}$ and $B_{2}$ goes to zero.

Therefore, we propose not to estimate the rare event probability $\gamma=\mathbb{P}\left(\tau_{B}<\zeta \mid X_{0}=x_{0}\right)$ using $h_{12}$ in (8) for disconnected $B$. Instead, we write $\gamma=\gamma_{1}+\gamma_{2}$ with

$$
\begin{aligned}
& \gamma_{1}:=\mathbb{P}\left(\tau_{B_{1}}<\zeta \mid X_{0}=x_{0}\right), \\
& \gamma_{2}:=\mathbb{P}\left(\tau_{B_{2}}<\zeta \wedge \tau_{B_{1}} \geq \zeta \mid X_{0}=x_{0}\right) .
\end{aligned}
$$

We can interpret this as the rare event being partitioned into a part where $B_{1}$ is hit in due time (and maybe $B_{2}$ as well), and a part where $B_{2}$ is hit in due time while $B_{1}$ is never hit during $[0, \zeta]$. We propose to separately estimate $\gamma_{1}$ and $\gamma_{2}$ using two independent splitting simulations. To obtain an unbiased estimator 
for $\gamma_{1}$ we simply choose $h_{1}$ in (9) as importance function (see Figure 4). To obtain an unbiased estimator for $\gamma_{2}$ we choose $h_{2}$ in (9) as importance function and a modified splitting framework rewarding sample paths approaching $B_{2}$ while killing those that hit $B_{1}$ (see Figure 5). We call these two runs together the separated splitting technique, as opposed to the single standard splitting technique using importance function $h_{12}$ in (8).

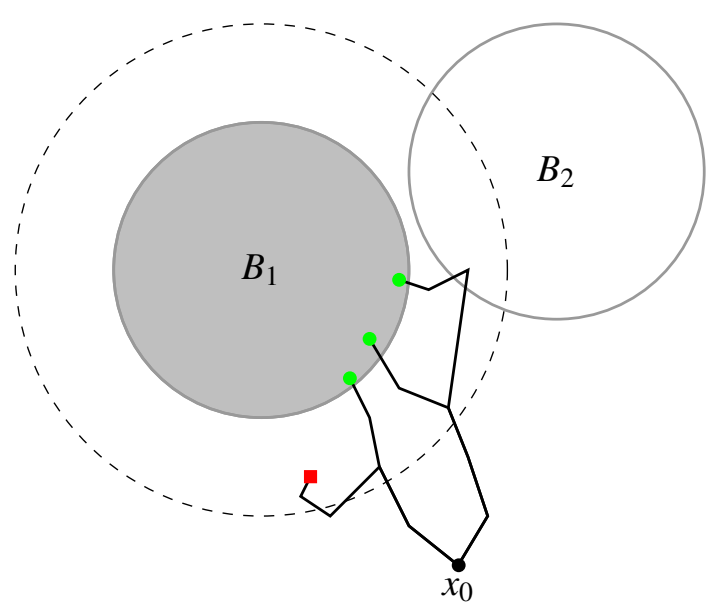

Figure 4: Illustration of splitting sample paths to estimate $\gamma_{1}=\mathbb{P}\left(\tau_{B_{1}}<\zeta \mid X_{0}=x_{0}\right)$, ignoring $B_{2}$. Green circles correspond to subpaths for which the rare event does occur, red squares correspond to subpaths for which it does not.

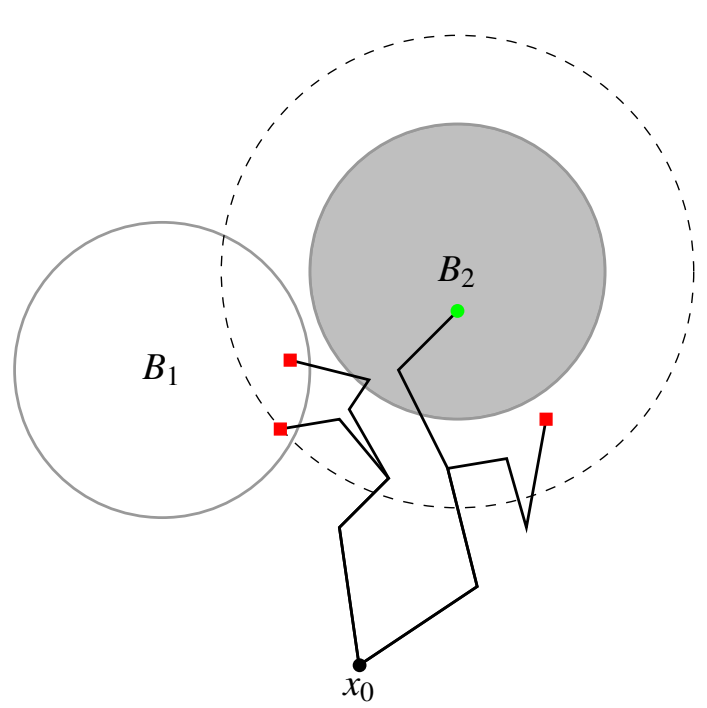

Figure 5: Illustration of splitting sample paths to estimate $\gamma_{2}=\mathbb{P}\left(\tau_{B_{2}}<\zeta \wedge \tau_{B_{1}} \geq \zeta \mid X_{0}=x_{0}\right)$, killing paths that enter $B_{1}$. Green circles correspond to subpaths for which the rare event does occur, red squares correspond to subpaths for which it does not.

More precisely, to estimate $\gamma_{2}$ we start from a standard splitting setup for $\mathbb{P}\left(\tau_{B_{2}}<\zeta \mid X_{0}=x_{0}\right)$ as described in subsection 2.2. We set $l_{k}, T_{k}$ and $D_{k}$ for all $k$ as before, but we modify the levels by defining

$$
\begin{aligned}
\bar{D}_{k} & :=D_{k} \cap\left\{\tau_{B_{1}} \geq T_{k}\right\} \quad \forall k=0, \ldots, m, \\
\bar{D}_{m+1} & :=D_{m} \cap\left\{\tau_{B_{1}} \geq \zeta\right\} .
\end{aligned}
$$

So to enter level $k$, we additionally require the chain not to have entered $B_{1}$ in the mean time. Further, the occurrence of the additional level $\bar{D}_{m+1}$ requires that after entering $B_{2}$ in due time, the chain should not have entered $B_{1}$ during the remaining time domain $\left[T_{m}, \zeta\right]$. Note that $\bar{D}_{m+1}$ is exactly the rare event, and since again $\bar{D}_{0} \supset \bar{D}_{1} \supset \cdots \supset \bar{D}_{m} \supset \bar{D}_{m+1}$ we can write

$$
\gamma_{2}=\mathbb{P}\left(\bar{D}_{m+1}\right)=\prod_{k=1}^{m+1} \mathbb{P}\left(\bar{D}_{k} \mid \bar{D}_{k-1}\right) .
$$

We may now resume the standard splitting technique as described in subsection 2.2 using $D_{k}=\bar{D}_{k}$ for all $k$. Therefore, an unbiased estimator for $\gamma_{2}$ is given by

$$
\hat{\gamma}_{2}:=\prod_{k=1}^{m+1} \hat{p}_{k},
$$


where for all levels $k \hat{p}_{k}$ is the proportion of sample paths for which $\bar{D}_{k}$ occurs. We conclude that $\hat{\gamma}_{1}+\hat{\gamma}_{2}$ is an unbiased estimator for $\gamma$. Furthermore, if assumption (4) holds we can compute bounds for the squared relative errors $\operatorname{SRE}\left(\hat{\gamma}_{1}\right)$ and $\operatorname{SRE}\left(\hat{\gamma}_{2}\right)$ using (5). Then a bound for $\hat{\gamma}_{1}+\hat{\gamma}_{2}$ follows immediately, since

$$
\operatorname{SRE}\left(\hat{\gamma}_{1}+\hat{\gamma}_{2}\right)=\frac{\operatorname{Var} \hat{\gamma}_{1}+\operatorname{Var} \hat{\gamma}_{2}}{\left(\gamma_{1}+\gamma_{2}\right)^{2}} \leq \frac{\operatorname{Var} \hat{\gamma}_{1}}{\gamma_{1}^{2}}+\frac{\operatorname{Var} \hat{\gamma}_{2}}{\gamma_{2}^{2}}=\operatorname{SRE}\left(\hat{\gamma}_{1}\right)+\operatorname{SRE}\left(\hat{\gamma}_{2}\right) .
$$

We used independence of $\gamma_{1}$ and $\gamma_{2}$ in the first equality.

\section{EXPERIMENT}

We compare the proposed separated splitting technique using importance functions $h_{1}$ and $h_{2}$ in (9) with the standard splitting technique using importance function $h_{12}$ in (8). The state space $\mathcal{X}=\mathbb{R}$ is one-dimensional and the rare event set $B=B_{1} \cup B_{2}$ consists of two separate intervals $B_{1}=\left[b_{1}, \infty\right)$ and $B_{2}=\left(-\infty, b_{2}\right], b_{1}>b_{2}$. We assume that $\left\{X_{t}, t \geq 0\right\}$ is a modification of an Ornstein-Uhlenbeck process, where we change the sign of the long-term mean whenever the process passes zero:

$$
X(t)=\theta\left(\mu \operatorname{sgn}\left(X_{t}\right)-X_{t}\right) d t+\sigma d W(t) .
$$

The drift of this stochastic differential equation corresponds to the potential

$$
V(x)=-\int \theta(\mu \operatorname{sgn}(x)-x) d x=\frac{\theta}{2}(x-\mu \operatorname{sgn}(x))^{2} .
$$

The resulting invariant distribution $\rho(x)$ of the process is proportional to

$$
\rho(x) \propto e^{-2 V(x) / \sigma^{2}}=\exp \left\{-\frac{\theta}{\sigma^{2}}(x-\mu \operatorname{sgn}(x))^{2}\right\} .
$$

Figure 6 shows that the double well potential (12) is just the original Ornstein-Uhlenbeck potential, mirrored in the y-axis. Similarly, Figure 7 shows that the bimodal stationary distribution (13) is just the normal distibution mirrored in the $\mathrm{y}$-axis, and then normalized.

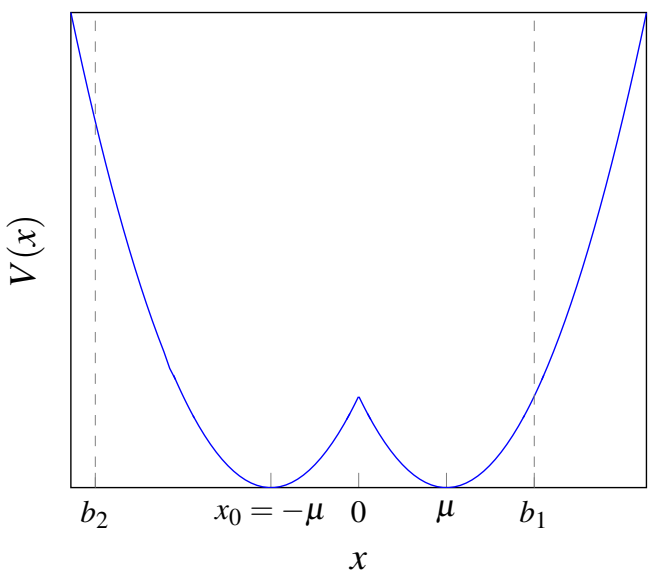

Figure 6: The double well potential (12) of the modified OU process in (11)

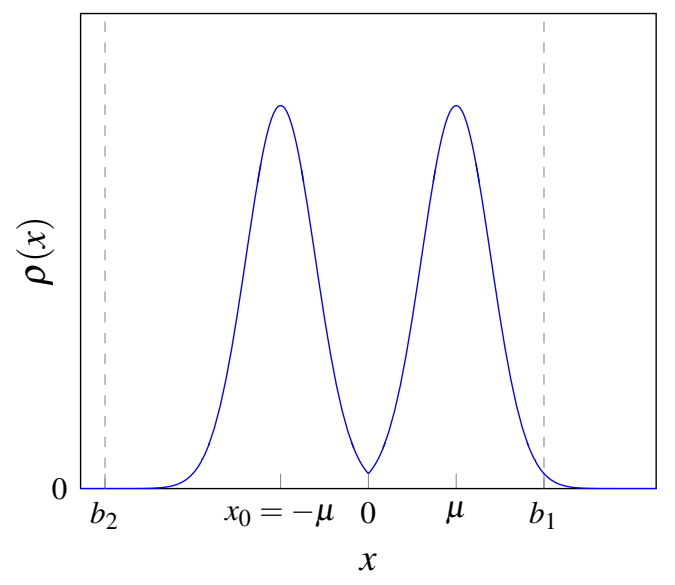

Figure 7: The bimodal stationary distribution (13) of the modified OU process in (11)

We substitute $\mu \operatorname{sgn}\left(X_{t}\right)$ for $\mu$ in the exact solution of the Ornstein-Uhlenbeck process (Gillespie 1996) to obtain a discretization of (11):

$$
X_{i+1}=X_{i} e^{-\theta \Delta}+\mu \operatorname{sgn} X_{i}\left(1-e^{-\theta \Delta}\right)+\sigma \sqrt{\frac{1-e^{-2 \theta \Delta}}{2 \theta}} Z_{i},
$$


with $X_{i}=X(i \Delta)$ with time step size $\Delta$, and $Z_{i} \sim N(0,1)$ i.i.d. standard normal for all $i$. We choose parameter values $\theta=\sigma=1, \mu=-x_{0}=2, b_{1}=4, b_{2}=-6, \Delta=0.001$ and a deterministic stopping time $\zeta=T=10$. We will estimate the rare event probability $\gamma=\mathbb{P}\left(\tau_{B}<T \mid X_{0}=x_{0}\right)$. Figure 6 shows that the process starts in the left well and initially has equal probability of going right or left. To arrive at $B_{1}$ the process should overcome the potential barrier $V(0)=2$ at $x=0$. However, the left and right tail masses in Figure 7 show that the steady state probability of $B_{1}$ is larger than that of $B_{2}$. In fact, the CMC estimates in Table 1 show that indeed $\gamma_{1}=\mathbb{P}\left(\tau_{B_{1}}<T \mid X_{0}=x_{0}\right)$ is much larger than $\gamma_{2}=\mathbb{P}\left(\tau_{B_{2}}<T \wedge \tau_{B_{1}} \geq \zeta \mid X_{0}=x_{0}\right)$. We regard these CMC estimates as reference values for splitting estimates.

Table 1: CMC estimates $\tilde{\gamma}_{n}$ for the subset probabilities, using $n=10^{7}$ samples

\begin{tabular}{l|cc} 
Probability & CMC estimate $\tilde{\gamma}_{n}$ & $\operatorname{SRE}\left(\tilde{\gamma}_{n}\right)$ \\
\hline$\gamma=\gamma_{1}+\gamma_{2}$ & $3.50 \times 10^{-3}$ & $2.85 \times 10^{-5}$ \\
$\gamma_{1}$ & $3.50 \times 10^{-3}$ & $2.85 \times 10^{-5}$ \\
$\gamma_{2}$ & $2.50 \times 10^{-6}$ & $4.00 \times 10^{-2}$
\end{tabular}

We use both the standard splitting technique as the separated splitting technique to estimate $\gamma$. We choose the levels as described in Section 3.1 and use $r_{k}=r=100$ hits per level. The standard splitting estimator for $\gamma$ is denoted $\hat{\gamma}_{12}$, whereas the separated splitting technique computes estimators $\hat{\gamma}_{1}$ for $\gamma_{1}$ and $\hat{\gamma}_{2}$ for $\gamma_{2}$, yielding $\hat{\gamma}_{1}+\hat{\gamma}_{2}$ for $\gamma$. We investigate both techniques by comparing their estimates with the CMC estimates. After repeating both simulations 1000 times we check if the squared relative errors $\operatorname{SRE}\left(\hat{\gamma}_{12}\right)$, $\operatorname{SRE}\left(\hat{\gamma}_{1}\right)$ and $\operatorname{SRE}\left(\hat{\gamma}_{2}\right)$ are indeed bounded by (5), which should hold under assumption (4).

Table 2 shows that the squared relative error $\operatorname{SRE}\left(\hat{\gamma}_{12}\right)$ of the standard splitting estimator is more than twice as high as the bound suggested by (5). This suggests that assumption (4) that the next level probability $p_{k}$ is independent of the entrance state $\left(X_{T_{k}}, T_{k}\right)$ does not hold. Table 3 shows that this is indeed the case: the number of hits on the left and right side levels of the importance function $h_{12}$ are listed per level. The number of hits at the left side level are significant at the first and second level, whereas the top level (and thus the rare event set) is only hit on the right side. We conclude that the next level probability depends significantly on the current level entrance state using the standard splitting technique.

Table 2: Standard splitting estimator $\hat{\gamma}_{12}$ for $\gamma$ and separated splitting estimator $\hat{\gamma}_{1}+\hat{\gamma}_{2}$ for $\gamma$. The squared relative error $\operatorname{SRE}\left(\hat{\gamma}_{12}\right)$ exceeds bound (5), whereas $\operatorname{SRE}\left(\hat{\gamma}_{1}\right)$ and $\operatorname{SRE}\left(\hat{\gamma}_{2}\right)$ do not.

\begin{tabular}{l|ccc} 
& Value & $\operatorname{SRE}(\cdot)$ & Bound for $\operatorname{SRE}(\cdot)$ \\
\hline$\hat{\gamma}_{12}$ & $2.48 \times 10^{-3}$ & 0.069 & 0.031 \\
$\hat{\gamma}_{1}$ & $3.58 \times 10^{-3}$ & 0.034 & 0.041 \\
$\hat{\gamma}_{2}$ & $1.52 \times 10^{-6}$ & 0.066 & 0.096
\end{tabular}

Table 3: Efficiency loss: at the first level a significant number of succesful sample paths approached the left interval 'in vain', since no sample path entered the left interval at the final level.

\begin{tabular}{l|cc} 
Level & Hits on left side & Hits on right side \\
\hline 1 & 67 & 33 \\
2 & 8 & 92 \\
3 & 0 & 100 \\
4 & 0 & 100
\end{tabular}

Separated splitting is not affected by this problem, and indeed the squared relative errors $\operatorname{SRE}\left(\hat{\gamma}_{1}\right)$ and $\operatorname{SRE}\left(\hat{\gamma}_{2}\right)$ do fulfill bound (5) in Table 2. Using (10) we find the bound $0.041+0.096=0.137$ for $\operatorname{SRE}\left(\hat{\gamma}_{1}+\hat{\gamma}_{2}\right)$. Assuming normality one can show that the implied conservative $95 \%$ confidence interval 


\section{Wadman, Crommelin, and Frank}

for $\gamma$ overlaps with the narrow CMC confidence interval. However, one can show that the conservative confidence interval implied by the standard splitting technique is a significant underestimation.

We repeat the experiment for higher barriers by increasing $\mu$, i.e. for smaller rare event probabilities. Table 4 shows that for barrier height $V(0)=2.42, \operatorname{SRE}\left(\hat{\gamma}_{12}\right)$ is 3 times as high as bound (5) suggests. Table 5 and 6 show that $\operatorname{SRE}\left(\hat{\gamma}_{12}\right)$ exceeds the theoretical bound (5) by a factor up to 15 for even higher barriers. This illustrates that the accuracy of the splitting estimate may be much lower than expected when one does not assess separated rare event subsets in separated splitting simulations.

Table 4: Experiment as in Table 2, but now $\mu=x_{0}=2.2$, thus raising the barrier height to $V(0)=2.42$, and $b_{1}=4.4, b_{2}=-6.6$. The squared relative error $\operatorname{SRE}\left(\hat{\gamma}_{12}\right)$ exceeds bound (5), whereas $\operatorname{SRE}\left(\hat{\gamma}_{1}\right)$ and $\operatorname{SRE}\left(\hat{\gamma}_{2}\right)$ do not.

\begin{tabular}{l|ccc} 
& Value & $\operatorname{SRE}(\cdot)$ & Bound for $\operatorname{SRE}(\cdot)$ \\
\hline$\hat{\gamma}_{12}$ & $5.28 \times 10^{-4}$ & 0.120 & 0.041 \\
$\hat{\gamma}_{1}$ & $8.75 \times 10^{-4}$ & 0.043 & 0.052 \\
$\hat{\gamma}_{2}$ & $6.18 \times 10^{-8}$ & 0.095 & 0.118
\end{tabular}

Table 5: Experiment as in Table 2, but now $\mu=x_{0}=2.5$, further raising the barrier height to $V(0)=3.125$, and $b_{1}=5, b_{2}=-6.5$. The squared relative error $\operatorname{SRE}\left(\hat{\gamma}_{12}\right)$ exceeds bound (5) by a factor 5 .

\begin{tabular}{l|ccc} 
& Value & $\operatorname{SRE}(\cdot)$ & Bound for SRE $(\cdot)$ \\
\hline$\hat{\gamma}_{12}$ & $2.56 \times 10^{-5}$ & 0.43 & 0.085 \\
$\hat{\gamma}_{1}$ & $3.91 \times 10^{-5}$ & 0.076 & 0.074 \\
$\hat{\gamma}_{2}$ & $1.79 \times 10^{-6}$ & 0.071 & 0.096
\end{tabular}

Table 6: Experiment as in Table 2, but now $\mu=x_{0}=3$, further raising the barrier height to $V(0)=4.5$, and $b_{1}=6, b_{2}=-7.2$. The squared relative error $\operatorname{SRE}\left(\hat{\gamma}_{12}\right)$ exceeds bound (5) by a factor 15 .

\begin{tabular}{l|ccc} 
& Value & $\operatorname{SRE}(\cdot)$ & Bound for $\operatorname{SRE}(\cdot)$ \\
\hline$\hat{\gamma}_{12}$ & $3.89 \times 10^{-7}$ & 1.42 & 0.096 \\
$\hat{\gamma}_{1}$ & $2.16 \times 10^{-7}$ & 0.121 & 0.107 \\
$\hat{\gamma}_{2}$ & $3.01 \times 10^{-7}$ & 0.078 & 0.107
\end{tabular}

Finally, we should note that $\operatorname{SRE}\left(\hat{\gamma}_{1}\right)$ is exceeding its bound as well in these last two experiments, although only to a moderate extent. To explain this, we point out that bound (5) is based on the product of the inequality

$$
\operatorname{SRE}\left(\hat{p}_{k}\right) \leq \frac{1}{r_{k}-2}
$$

over all levels $k$, where $r_{k}$ is the number of hits at level $k$. The simulation results show that every transgression of the bound by $\operatorname{SRE}\left(\hat{\gamma}_{1}\right)$ corresponds to violation of (14) for the level for which $X_{t}$ has to traverse the interval $[0, \mu]$. For large $\mu$ the drift $-\nabla V\left(X_{t}\right)$ of $X_{t}$ rapidly changes at left side of this interval (see Figure $6)$. Hence even for a small time step the probability that $X_{t}$ enters the next level depends sensitively on the entrance state of the previous level, whereby we cannot assume (4) anymore. Nevertheless, the overshoot of $\operatorname{SRE}\left(\hat{\gamma}_{1}\right)$ is a factor 1.03 and 1.14 , respectively, which is small compared to that of $\operatorname{SRE}\left(\hat{\gamma}_{12}\right)$ of a factor 5 and 15 , respectively. 
Wadman, Crommelin, and Frank

\section{CONCLUSION}

Estimating the probability of a rare event set consisting of multiple separated subsets by use of a splitting technique is challenging since the most likely path to the rare event set may be very different from the most likely path to an intermediate level. We proposed a separated splitting technique to mitigate the difficulties posed by this path deviation by estimating the probability of hitting each subset individually. For each subset a modified splitting simulation replicates samples paths that approach this subset, while killing those that enter a subset that is already addressed in a previous simulation.

We compared the separated splitting technique with a standard splitting technique by estimating the probability of entering either of two separated intervals on the real line. Choosing a stochastic process with a potential barrier close to one of the intervals, we show that the standard splitting technique replicates sample paths that approach the subset that is not reached in the end. This efficiency loss - quantified as the squared relative error of the estimator - is observed to be significantly higher when using standard splitting than when using separated splitting. This difference is shown to become more severe for smaller rare event probabilities, illustrating the advantage of the separated splitting technique.

\section{REFERENCES}

Amrein, M., and H. R. Künsch. 2011. "A Variant of Importance Splitting for Rare Event Estimation: Fixed Number of Successes". ACM Transactions on Modeling and Computer Simulation 21 (2): 13.

Billinton, R., and W. Li. 1994. Reliability Assessment of Electrical Power Systems using Monte Carlo Methods. Springer.

de Boer, P.-T. 2000. Analysis and efficient simulation of queueing models of telecommunication systems. $\mathrm{Ph}$. D. thesis.

Dean, T., and P. Dupuis. 2009. "Splitting for rare event simulation: A large deviation approach to design and analysis". Stochastic processes and their applications 119 (2): 562-587.

Del Moral, P., and J. Garnier. 2005. "Genealogical particle analysis of rare events". The Annals of Applied Probability 15 (4): 2496-2534.

Garvels, M. 2000. The Splitting Method in Rare Event Simulation. Ph. D. thesis, Universiteit Twente.

Garvels, M. J., J.-K. C. Van Ommeren, and D. P. Kroese. 2002. "On the importance function in splitting simulation". European Transactions on Telecommunications 13 (4): 363-371.

Gillespie, D. T. 1996. "Exact Numerical Simulation of the Ornstein-Uhlenbeck Process and its Integral". Physical review E 54 (2): 2084.

Glasserman, P., P. Heidelberger, P. Shahabuddin, and T. Zajic. 1998. "A large deviations perspective on the efficiency of multilevel splitting". Automatic Control, IEEE Transactions on 43 (12): 1666-1679.

Glasserman, P., P. Heidelberger, P. Shahabuddin, and T. Zajic. 1999. "Multilevel Splitting for Estimating Rare Event Probabilities". Operations Research 47 (4): 585-600.

Grainger, J., and W. Stevenson. 1994. Power system analysis, Volume 621. McGraw-Hill.

L'Ecuyer, P., V. Demers, and B. Tuffin. 2006. "Splitting for Rare-Event Simulation". In Proceedings of the Winter Simulation Conference 2006, 137-148. IEEE.

Maragliano, L., A. Fischer, E. Vanden-Eijnden, and G. Ciccotti. 2006. "String method in collective variables: minimum free energy paths and isocommittor surfaces". The Journal of chemical physics 125 (2): 024106.

Rubino, G., and B. Tuffin. 2009. Rare Event Simulation using Monte Carlo Methods. John Wiley \& Sons.

Vanden-Eijnden, E., and M. Venturoli. 2009. "Exact rate calculations by trajectory parallelization and tilting". The Journal of chemical physics 131 (4): 044120.

Villén-Altamirano, M., and J. Villén-Altamirano. 2006. "On the efficiency of RESTART for multidimensional state systems". ACM Transactions on Modeling and Computer Simulation (TOMACS) 16 (3): 251-279.

Wadman, W., D. Crommelin, and J. Frank. 2013. "Applying a Splitting Technique to Estimate Electrical Grid Reliability”. In Winter Simulation Conference 2013. 
Wang, S.-P., A. Chen, C.-W. Liu, C.-H. Chen, and J. Shortle. 2011. "Rare-Event Splitting Simulation for Analysis of Power System Blackouts". In Power and Energy Society General Meeting, 2011 IEEE, 1-7. IEEE.

\begin{abstract}
AUTHOR BIOGRAPHIES
WANDER WADMAN is a PhD student in the Scientific Computing group at CWI Amsterdam. He received a master's degree in Applied Mathematics at Delft University of Technology. His PhD topic is the stochastic modeling of electric power systems, and his research interests include stochastic modeling of dynamical systems, power flow problems and Monte Carlo methods with a focus on rare event simulation. E-mail: w.wadman@cwi.nl, homepage: www.cwi.nl/ wadman/.
\end{abstract}

DAAN CROMMELIN is professor of Numerical Analysis and Dynamical Systems at the University of Amsterdam. He received his $\mathrm{PhD}$ from Utrecht University in 2003 and worked as a postdoc at New York University's Courant Institute. Since 2006 he is a researcher at CWI Amsterdam where he is now leader of the Scientific Computing group. His research focuses on stochastic methods and statistical inference for dynamical systems, with applications in atmosphere-ocean science and energy systems. E-mail: daan.crommelin@cwi.nl, homepage: www.cwi.nl/ dtc.

JASON FRANK is professor of Numerical Analysis at Utrecht University. He received his $\mathrm{PhD}$ in numerical analysis from Delft University of Technology. From 2000 to 2013 we was employed by CWI Amsterdam as researcher and (from 2007) leader of the research group Computational Dynamics. From 2010 to 2013 he held the chair of Numerical Analysis and Dynamical Systems at the University of Amsterdam. His research addresses geometric numerical methods for partial differential equations, with applications in geophysical fluid dynamics and nonlinear waves. E-mail: j.e.frank@uu.nl, homepage: www.staff.science.uu.nl/ frank011/. 